\title{
Diagnostic and Management of Undescended Ovary - A Preoperative Dilemma: A Case-Based Systematic Review
}

\author{
Ioana Anca Stefanopol 1,2,* \\ Liliana Baroiu ${ }^{3,4}$ \\ Georgiana Bianca Constantin ${ }^{1,5}$ \\ Dumitru Marius Danila 2,6,* \\ Lucreția Anghel (iD) $3,7, *$ \\ Alexandru Nechifor ${ }^{3,8, *}$ \\ Alin Laurențiu Tatu (D) $3,8-10$ \\ 'Department of Morphological and Functiona \\ Sciences, Faculty of Medicine and Pharmacy, \\ "Dunărea de Jos" University, Galați, Romania; \\ ${ }^{2}$ Department of Pediatric Surgery and Orthopedy, \\ Clinical Emergency Hospital for Children "Sf loan", \\ Galati, Romania; ${ }^{3}$ Clinical Medical Department, \\ Faculty of Medicine and Pharmacy, "Dunărea de \\ Jos" University, Galați, Romania; ${ }^{4}$ Infectious \\ Diseases Department, Clinical Hospital of \\ Infectious Diseases "St. Parascheva”, Galați, \\ Romania; ${ }^{5}$ West Clinique Braila, Braila, Romania; \\ ${ }^{6}$ Clinical Surgical Department, Faculty of Medicine \\ and Pharmacy, "Dunărea de Jos" University, Galați, \\ Romania; ${ }^{7}$ Internal Medicine Department, "St. \\ Andrew the Apostle" County Emergency Clinical \\ Hospital, Galați, Romania; ${ }^{8}$ Multidisciplinary \\ Integrated Center of Dermatological Interface \\ Research, "Dunărea de Jos" University, Galați, \\ Romania; 'Research Center in the Field of Medical \\ and Pharmaceutical Sciences, ReFORM-UDJ, \\ Galați, Romania; ${ }^{10}$ Dermatology Department, \\ Clinical Hospital of Infectious Diseases "Sf. \\ CuvioasaParascheva”, Galati, Romania \\ *These authors contributed equally to this work
}

Correspondence: Liliana Baroiu

Clinical Medical Department, Faculty of

Medicine and Pharmacy, "Dunărea de Jos"

University, 47 Domnească Street, Galati,

800010 , Romania

Tel +4072320l24l

Email lilibaroiu@yahoo.com

Georgiana Bianca Constantin

Department of Morphological and

Functional Sciences, Faculty of Medicine

and Pharmacy, "Dunărea de Jos"

University, 47 Domnească Street, Galați,

800010 , Romania

Tel +40749058284

Email constantin_bianca2009@yahoo.com
Abstract: Undescended ovary (UO) is an uncommon congenital condition characterized by the presence of the adnexa above the common iliac vessels, with an estimated incidence of $0.3-2 \%$. Because of its rarity, it is usually presented as a case report. A thorough knowledge of the ovarian embryological development is essential for the clinician, who must be warned about the possibility of associated Müllerian and renal malformations. There may be asymptomatic patients, incidentally diagnosed during infertility evaluation, but when symptoms occur, these are unspecific and most often this disorder is misdiagnosed, the accurate diagnosis being established intraoperatory. The malignant potential of an UO is accepted, although no such cases were reported. The role of the UO in infertility is still unclear, despite evidence of its normal function. Complications are linked to the ovary (cyst formation, cyst ruptures or tumors) or to the undescended fallopian tube (ectopic pregnancies). The management should be conservative, but there is no consensus about whether it is necessary to excise the ipsilateral undescended tube. We included a short case presentation of an UO cystadenoma misdiagnosed as a renal cyst, which depicts all diagnostic and management dilemmas and inspired us to write this review. The present literature review includes all the cases reported from the early 20th century to the present, with updated data about epidemiology, pathophysiology, clinical and imaging diagnosis, treatment options and prognosis of this rare condition. This paper aims to establish some directions in the diagnosis and management of similar patients and to remind us that, no matter how advanced the imaging investigation techniques might be, a correct preoperative diagnosis may often be missed.

Keywords: undescended ovary, ovarian maldescent, undescended fallopian tubes, maldescent fallopian tube, ovarian cyst, ectopic tubal pregnancy

\section{Introduction}

Undescended ovary (UO), defined by the presence of the superior adnexal pole above common iliac vessels, is a rare gynecological condition, usually found as case reports in medical publications. Due to the common embryological origin of both genital and renal tract, UO frequently occurs in association with Müllerian and renal malformations. ${ }^{1-3}$ It is difficult to establish the diagnosis of UO based on clinical signs and symptoms, due to their lack of specificity, or because most the patients $(60 \%)$ are asymptomatic. ${ }^{4-6}$ In the asymptomatic cases, UO is incidentally discovered during the investigations for infertility. ${ }^{7}$ Usually, only complicated UO are symptomatic. Complications may be linked to the ovary (cyst formation, cyst 
ruptures or tumors) or to the undescended noncommunicating fallopian tube (4\% risk of ectopic pregnancies). These cases are often misdiagnosed, physicians rather considering other more frequent etiologies of the acute abdominal pain in female patients, such as acute appendicitis, corpus luteum cyst rupture, ectopic pregnancy or ovarian torsion. ${ }^{6,8}$ Imaging investigations such as ultrasonography (USG), hysterosalpingogram (HSG) or magnetic resonance imaging (MRI) are more useful to an accurate diagnosis. ${ }^{9,10}$ But even these advanced techniques are sometimes ineffective, and the UO is discovered during laparotomy or laparoscopy. ${ }^{11,12}$ As undescended ovaries have a normal function if there are no malignant features, the management should be conservative. ${ }^{13-15}$ The malignant potential of an UO is accepted, although no such cases have been reported. ${ }^{16,17}$ The role of the UO in infertility is still unclear. ${ }^{2,18}$ The aim of the present review is to provide a complete and accurate picture of this rare pathology, including documented cases from the early 20th century to the present. We classified the cases according to the presence of symptoms and age of the patients, hoping to capture specific aspects useful for other colleagues facing the same pathology and for establishing some directions in the diagnosis and management of similar cases.

\section{Methods}

A literature review was performed to identify all relevant medical papers from Medline, PubMed, Citationsy Archives and ResearchGate databases focused on the following keywords in different combinations: undescended ovary, ovarian maldescent, undescended fallopian tubes, maldescent fallopian tube, ovarian cyst, ectopic tubal pregnancy. Only the articles written in English were selected. The references of the initially identified publications and the similar articles were assessed and eventually added to the present review. In addition, observations gathered from the medical literature were supplemented with results and practical conclusions obtained through authors' personal research. Our searches yielded a total of 184 articles, but only 66 formed the basis of this review, as shown in the PRISMA flow diagram of the selection process (Figure 1).

The vast majority $(n=48)$ are case reports, four of them including some data from literature review. We found only 2 mini-reviews, but these were quite incompletely documented. For instance, Altinay-Kirli et al in 2017 reported 4 cases of pediatric patients but our research found that by 2017 there were 9 such cases. ${ }^{11}$ We gathered data about epidemiology, pathophysiology, clinical and imaging diagnosis, complications, treatment options and prognosis of this rare condition. Our research findings were grouped into 3 tables containing useful information for an accurate diagnosis and management (Table 1: pediatric patients, Table 2: symptomatic adult patients and Table 3: asymptomatic adult patients). For an easier understanding of this review's results, we decided to write a paragraph of discussions at the end of each section.

\section{Definition and Terminology Issues}

The first classification system of an abnormal located ovary was created by Wharton in $1959 .^{19}$ There were two groups: accessory (ovarian tissue located near and connected to a normally placed ovary) and supernumerary (at a distance from normal ovaries). Because this classification supposed the presence of both normal ovaries and did not include the ovarian implant syndrome, in 1991 Lachman and Berman suggested the division into three groups: post-surgical implant, post-inflammatory implant, and true ectopic (embryologic). ${ }^{20}$ Since then, UO is considered to be a congenital condition occurring when its descent is arrested in the retroperitoneal space between the lumbar region and its normal position in the ovarian fossa. Ectopic ovaries may also be found in other abnormal sites such as omentum, mesentery, inguinal canal, or attached to the uterus or bladder, ${ }^{15}$ as a consequence of the autoamputation of the ovary followed by autotransplantation in other sites. ${ }^{21,22}$

The pelvic brim was initially considered to be the landmark for UO ${ }^{23,24}$ In a study based on pelvic MRI examinations, Allen et al used the pelvic brim (a line from the superior margin of the symphysis pubis to the sacral promontory) as the imaging landmark for analysis. The incidence of UO was higher compared to the one found when the landmark was the common iliac artery bifurcation. Hence, in correlation with prior studies, they considered the second landmark for defining incomplete ovarian descent. ${ }^{9}$

\section{Embryology}

Both urinary and genital systems develop from the intermediate mesoderm located on each side of the spine on the posterior wall of the abdomen. During the 4th intrauterine week in the thoraco-lumbar region the mesonephros and the mesonephric or Wolffian ducts will develop. ${ }^{25}$ In the 5 th week, on the medial side of the mesonephros appears the genital or gonadal ridge, by proliferation of the coelomic epithelium and the underlying mesenchyme. ${ }^{26}$ At the same time develop the paramesonephric or Müllerian ducts as an invagination of the coelomic epithelium lateral to the Wolffian ducts. ${ }^{27}$ The 


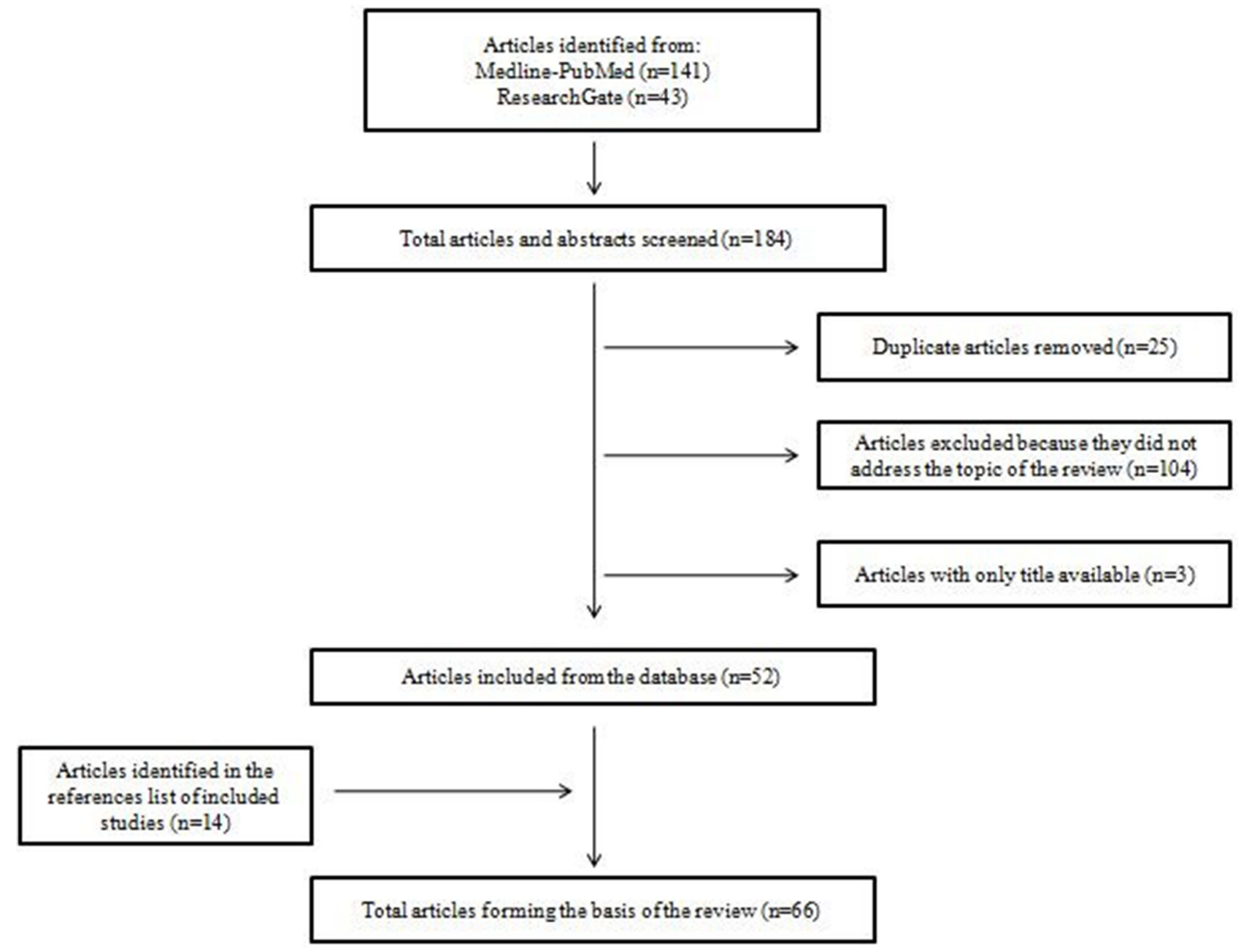

Figure I PRISMA flow diagram of search and study selection process.

Note: PRISMA figure adapted from Liberati A, Altman D, Tetzlaff J, et al. The PRISMA statement for reporting systematic reviews and meta-analyses of studies that evaluate health care interventions: explanation and elaboration. Journal of clinical epidemiology. 2009;62(10). Creative Commons. ${ }^{67}$

cephalic segments of the Müllerian ducts will form the fallopian tubes, while the distal parts fuse at the end of the second fetal month to form the uterus, cervix and the upper part of the vagina. In the third week primordial germ cells form in the endoderm of the posterior wall of the yolk sac, near the allantois. They will migrate toward the genital ridge, where they arrive in the 6th week, forming the indifferent gonad. ${ }^{25,26}$ In the absence of the SRY gene (sex-determining region on the Y chromosome), females produce estrogen instead of testosterone and do not produce anti-Müllerian factor. Consequently, the Wolffian ducts degrade, the Müllerian ducts will form the female internal sex organs, and the indifferent gonad becomes ovary. The ovaries can be identified in the 10th week. The follicle formation starts at 10-11 weeks of pregnancy, and in the 16th week there already exist primordial follicles. ${ }^{26}$ During the 6th month will also appear secondary (preantral) follicles, and at the end of the third trimester of pregnancy some tertiary De Graaf follicles can be found. Starting from the third month of fetal life, the ovaries descend guided by the gubernaculum attached to the inferior pole. ${ }^{17}$ The cranial part of the gubernaculum forms the proper ovarian ligament and its caudal part becomes the round ligament of the uterus. ${ }^{28}$ The superior pole of the fetal ovary is attached to the cranial genital ligament, which becomes the infundibulopelvic ligament. ${ }^{14,29}$ In the 5 th fetal month the ovaries should be located in the iliac region and at birth they reach the pelvic inlet, but it is only during the immediate postpartum period that they complete the descent into the ovarian fossa. ${ }^{30,31}$

\section{Pathogenesis and Associated Malformations}

The underlying mechanism of ovarian maldescent is still to be elucidated. There are theories supporting an impaired 
Table I Cases in Pediatric Population

\begin{tabular}{|c|c|c|c|c|c|c|c|}
\hline Authors, Year & Laterality & $\begin{array}{c}\text { Müllerian } \\
\text { Malformations }\end{array}$ & $\begin{array}{c}\text { Renal } \\
\text { Malformations }\end{array}$ & $\begin{array}{l}\text { Methods of } \\
\text { Diagnosis }\end{array}$ & $\begin{array}{c}\text { Preoperative } \\
\text { Diagnosis }\end{array}$ & $\begin{array}{c}\text { Postoperative } \\
\text { Diagnosis }\end{array}$ & $\begin{array}{l}\text { Surgical } \\
\text { Treatment }\end{array}$ \\
\hline Pidcock et al, $1930^{43}$ & Bilateral & None & Not listed & Laparotomy & $\begin{array}{c}\text { Acute } \\
\text { appendicitis }\end{array}$ & Bilateral UO & Oophorectomy \\
\hline Walker et al, $1935^{43}$ & Right & None & Not listed & Laparotomy & $\begin{array}{c}\text { Acute } \\
\text { appendicitis }\end{array}$ & Right UO & Oophorectomy \\
\hline Rock et al, $1986^{23}$ & Bilateral & Cervix atresia & None & Laparotomy & Not listed & Bilateral UO & Not listed \\
\hline Campbell et al, $1988^{54}$ & Right & None & Not listed & Laparotomy & $\begin{array}{c}\text { Abdominopelvic } \\
\text { mass }\end{array}$ & Cystadenofibroma & Oophorectomy \\
\hline Trinidad et al, $2004^{4}$ & Left & MRKH & Left RA & MRI, USG & None & None & None \\
\hline Kives et al, $2004^{30}$ & Bilateral & $\begin{array}{l}\text { Bicornuate } \\
\text { uterus }\end{array}$ & None & $\mathrm{CT}$, laparoscopy & $\begin{array}{l}\text { Intestinal } \\
\text { duplication }\end{array}$ & $\begin{array}{l}\text { Ruptured ovarian } \\
\text { hemorrhagic cyst }\end{array}$ & None \\
\hline Mama et al, $2012^{65}$ & Right & Left UU & None & $\begin{array}{l}\text { Colonoscopy, CT, } \\
\text { USG, laparoscopy }\end{array}$ & $\begin{array}{c}\text { Acute } \\
\text { appendicitis }\end{array}$ & Right UO & Oophorectomy \\
\hline Das et al, $2014^{53}$ & Right & Left UU & None & $\begin{array}{l}\text { USG, MRI, } \\
\text { laparoscopy }\end{array}$ & $\begin{array}{l}\text { Intestinal } \\
\text { duplication }\end{array}$ & Serous cystadenoma & Cystectomy \\
\hline Suh et al, $2016^{5}$ & Right & Left UU & None & $\begin{array}{l}\text { USG, MRI, } \\
\text { laparoscopy }\end{array}$ & $\begin{array}{c}\text { Retroperitoneal } \\
\text { pseudocyst }\end{array}$ & $\begin{array}{l}\text { Ruptured ovarian } \\
\text { hemorrhagic cyst }\end{array}$ & Salpingectomy \\
\hline Sekmenli et al, $2017^{6}$ & Right & None & None & USG, CT, laparotomy & $\begin{array}{l}\text { Renal cyst, } \\
\text { mucocele }\end{array}$ & $\begin{array}{l}\text { Ruptured ovarian } \\
\text { hemorrhagic cyst }\end{array}$ & Cystectomy \\
\hline Kirli et al, $2017^{11}$ & Right & Left UU & $\begin{array}{l}\text { Right pelvic } \\
\text { kidney }\end{array}$ & $\begin{array}{l}\text { USG, MRI, } \\
\text { laparotomy }\end{array}$ & Duplication cyst & Right UO & $\begin{array}{l}\text { Ovarian } \\
\text { fixation }\end{array}$ \\
\hline Present case & Right & Left UU & Right RA & $\begin{array}{l}\text { USG, MRI, } \\
\text { laparotomy }\end{array}$ & $\begin{array}{c}\text { Retroperitoneal } \\
\text { (renal) cyst }\end{array}$ & Serous cystadenoma & $\begin{array}{l}\text { Salpingo- } \\
\text { ovariectomy }\end{array}$ \\
\hline
\end{tabular}

Abbreviations: MRKH, Mayer-Rokitansky-Küster-Hauser syndrome; UU, unicornuate uterus; RA, renal agenesis; UO, undescended ovary; USG, ultrasonography; CT, computed tomography; MRI, magnetic resonance imaging.

descent of the gonads into the true pelvis caused by the gubernaculum failure to attach to the inferior pole of the ovary. ${ }^{32,33}$ Another theory is a retarded differential growth of the urogenital ridge's specific region responsible for the formation of the ovaries and fallopian tubes. ${ }^{34,35}$

UO may occur in patients with normal uterus. However, although the ovarian embryological development follows a different path than the uterus and fallopian tubes, the UO incidence is higher in the presence of uterine anomalies, particularly the unicornuate uterus (UU) caused by a lack of development of one Müllerian duct or by a disturbed descent to its proper location. ${ }^{36,37}$ This fact supports the theory of a multifactorial polygenic process at the end of the second gestational month. ${ }^{8,11,14}$ Some claim that the abnormal ovarian migration could affect the paramesonefrotic fusion, ${ }^{4,32}$ while others hypothesize that the descent of the ovary depends on a proper development of the Müllerian system..$^{30,33}$
Due to the common embryological origin, UO may occur in conjunction with renal malformations such as agenesis, ectopic kidney, double renal pelvis and horseshoe kidneys. ${ }^{14,19,20}$

Other rare associations reported in the literature are appendicular or colonic anomalies, ${ }^{31,38}$ double inferior vena cava, ${ }^{35}$ and intestinal malrotation. ${ }^{11}$ Recently. an exceedingly rare association with dermatomyositis and myasthenia gravis was reported. ${ }^{39}$ In cases of autoimmune diseases, the diagnosis of UO is detected incidentally during a careful screening and, if those conditions require corticotherapy (dexamethasone), this therapy should be used with special care, like in postoperative care, and should not determine adverse effects. ${ }^{40,41}$

\section{Incidence}

Undescended ovary was described for the first time in 1926 by Evans and Cade, under the name "Ectopic 
Table 2 Symptomatic Cases in Adult Patients

\begin{tabular}{|c|c|c|c|c|c|c|c|}
\hline Authors, Year & Laterality & $\begin{array}{c}\text { Müllerian } \\
\text { Malformation }\end{array}$ & $\begin{array}{c}\text { Renal } \\
\text { Malformation }\end{array}$ & $\begin{array}{l}\text { Methods of } \\
\text { Diagnosis }\end{array}$ & $\begin{array}{c}\text { Preoperative } \\
\text { Diagnosis }\end{array}$ & $\begin{array}{c}\text { Postoperative } \\
\text { Diagnosis }\end{array}$ & $\begin{array}{c}\text { Surgical } \\
\text { Treatment }\end{array}$ \\
\hline $\begin{array}{l}\text { Evans et al, } \\
1925^{42}\end{array}$ & Right & Left UU & Not listed & LT & $\begin{array}{c}\text { Acute } \\
\text { appendicitis }\end{array}$ & Right UO & Ovariectomy \\
\hline $\begin{array}{l}\text { Walker et al, } \\
1935^{43}\end{array}$ & Right & None & Not listed & LT & $\begin{array}{c}\text { Acute } \\
\text { appendicitis }\end{array}$ & $\begin{array}{l}\text { Pyosalpinx } \\
\text { Right UO }\end{array}$ & $\begin{array}{c}\text { Salpingo- } \\
\text { ovariectomy }\end{array}$ \\
\hline $\begin{array}{l}\text { Adnopoz et al, } \\
\left.195\right|^{38}\end{array}$ & Left & None & Not listed & LT & $\begin{array}{l}\text { Left ovarian } \\
\text { cyst torsion }\end{array}$ & $\begin{array}{c}\text { Pseudomucinous } \\
\text { cystadenoma }\end{array}$ & Cystectomy \\
\hline $\begin{array}{l}\text { Nichols et al, } \\
1951^{52}\end{array}$ & Right & Left UU & Right RA & $\begin{array}{l}\text { Cystoscopy } \\
\text { IVP, LT }\end{array}$ & $\begin{array}{c}\text { Acute } \\
\text { cholecystitis }\end{array}$ & $\begin{array}{c}\text { Hemorrhagic } \\
\text { lutein cyst }\end{array}$ & Oophorectomy \\
\hline $\begin{array}{l}\text { Dabby et al, } \\
1977^{5}\end{array}$ & Right & Left UU & Right RA & IVP, LT & None & $\begin{array}{c}\text { Ectopic } \\
\text { pregnancy }\end{array}$ & Not listed \\
\hline $\begin{array}{l}\text { Granat et al, } \\
\left.198\right|^{46}\end{array}$ & Left & Right UU & Left RA & IVP, HSG LT & $\begin{array}{c}\text { Ectopic } \\
\text { pregnancy }\end{array}$ & $\begin{array}{c}\text { Ectopic } \\
\text { pregnancy }\end{array}$ & Not listed \\
\hline \multirow[t]{4}{*}{$\begin{array}{l}\text { Rock et al, } \\
1986^{23}\end{array}$} & Right & $\begin{array}{c}\text { Bicornuate } \\
\text { uterus }\end{array}$ & None & LT & Not listed & Not listed & Not listed \\
\hline & Left & Right UU & $\begin{array}{c}\text { Bilateral } \\
\text { malrotation }\end{array}$ & LT & Not listed & Not listed & Not listed \\
\hline & Left & Right UU & Left RA & LT & Not listed & Not listed & Not listed \\
\hline & Bilateral & Cervix atresia & None & LT & Not listed & Not listed & Not listed \\
\hline $\begin{array}{l}\text { Brown et al, } \\
1987^{24}\end{array}$ & Left & Right UU & Left RA & IVP, USG LT & $\begin{array}{c}\text { Ectopic } \\
\text { pregnancy }\end{array}$ & $\begin{array}{c}\text { Ruptured tubal } \\
\text { pregnancy }\end{array}$ & Salpingectomy \\
\hline $\begin{array}{l}\text { Seoud et al, } \\
1987^{5}\end{array}$ & Left & Right UU & $\begin{array}{l}\text { Left pelvic } \\
\text { kidney }\end{array}$ & LT & $\begin{array}{c}\text { Ectopic } \\
\text { pregnancy }\end{array}$ & $\begin{array}{c}\text { Ectopic } \\
\text { pregnancy }\end{array}$ & Not listed \\
\hline $\begin{array}{l}\text { Pokoli et al, } \\
1989^{5,64}\end{array}$ & Left & Right UU & None & HSG, LS & $\begin{array}{c}\text { Ectopic } \\
\text { pregnancy }\end{array}$ & $\begin{array}{c}\text { Ectopic } \\
\text { pregnancy }\end{array}$ & Not listed \\
\hline $\begin{array}{l}\text { Timmerman et al, } \\
1983^{62}\end{array}$ & Right & Left UU & Right RA & $\begin{array}{l}\text { IVP, TVUS, } \\
\text { LS }\end{array}$ & $\begin{array}{c}\text { Ectopic } \\
\text { pregnancy }\end{array}$ & $\begin{array}{c}\text { Ruptured tubal } \\
\text { pregnancy }\end{array}$ & Salpingectomy \\
\hline $\begin{array}{l}\text { Knudsen et al, } \\
1994^{63}\end{array}$ & Left & Right UU & Left RA & USG, LS & $\begin{array}{c}\text { Ectopic } \\
\text { pregnancy }\end{array}$ & $\begin{array}{c}\text { Ectopic } \\
\text { pregnancy }\end{array}$ & Salpingectomy \\
\hline $\begin{array}{l}\text { Cohen et al, } \\
2001^{12}\end{array}$ & Left & None & None & CT, LS & $\begin{array}{l}\text { Abdominal } \\
\text { hemangioma }\end{array}$ & Functional cyst & $\begin{array}{c}\text { Salpingo- } \\
\text { ovariectomy }\end{array}$ \\
\hline $\begin{array}{l}\text { Gabriel et al, } \\
2002^{5}\end{array}$ & Right & Left UU & Right RA & IVP, USG LS & $\begin{array}{c}\text { Ectopic } \\
\text { pregnancy }\end{array}$ & $\begin{array}{c}\text { Ectopic } \\
\text { pregnancy }\end{array}$ & Not listed \\
\hline $\begin{array}{l}\text { Watkins et al, } \\
2004^{61}\end{array}$ & Right & Left UU & Not listed & USG, CT LS & $\begin{array}{l}\text { Appendiceal } \\
\text { tumor }\end{array}$ & Right UO & None \\
\hline $\begin{array}{l}\text { Trinidad et al, } \\
2004^{4}\end{array}$ & Right & None & None & $\begin{array}{l}\text { HSG,MRI } \\
\text { TVUS, }\end{array}$ & None & Right UO & None \\
\hline $\begin{array}{l}\text { El-Bishry et al, } \\
2006^{48}\end{array}$ & Right & Left UU & None & IVP, USG LS & $\begin{array}{c}\text { Ectopic } \\
\text { pregnancy }\end{array}$ & $\begin{array}{c}\text { Ectopic } \\
\text { pregnancy }\end{array}$ & Salpingectomy \\
\hline
\end{tabular}

(Continued) 
Table 2 (Continued).

\begin{tabular}{|c|c|c|c|c|c|c|c|}
\hline Authors, Year & Laterality & $\begin{array}{c}\text { Müllerian } \\
\text { Malformation }\end{array}$ & $\begin{array}{c}\text { Renal } \\
\text { Malformation }\end{array}$ & $\begin{array}{l}\text { Methods of } \\
\text { Diagnosis }\end{array}$ & $\begin{array}{c}\text { Preoperative } \\
\text { Diagnosis }\end{array}$ & $\begin{array}{c}\text { Postoperative } \\
\text { Diagnosis }\end{array}$ & $\begin{array}{c}\text { Surgical } \\
\text { Treatment }\end{array}$ \\
\hline $\begin{array}{l}\text { Nielsen et al, } \\
2010^{58}\end{array}$ & Right & Left UU & None & IVP, USG LS & $\begin{array}{c}\text { Ectopic } \\
\text { pregnancy }\end{array}$ & $\begin{array}{c}\text { Ectopic } \\
\text { pregnancy }\end{array}$ & Salpingectomy \\
\hline $\begin{array}{l}\text { Desteli et al, } \\
2013^{31}\end{array}$ & Left & Right UU & None & USG, MRI LS & LUO cyst & Paraovarian cyst & Cystectomy \\
\hline $\begin{array}{l}\text { Kollia et al, } \\
2014^{29}\end{array}$ & Right & None & Right RA & USG, MRI LT & RUO & Right UO & Oophorectomy \\
\hline $\begin{array}{l}\text { Mahmoud et al, } \\
2014^{50}\end{array}$ & Right & Left UU & $\begin{array}{l}\text { Right pelvic } \\
\text { kidney }\end{array}$ & $\begin{array}{c}\text { USG, MRI } \\
\text { HSG }\end{array}$ & RUO & None & None \\
\hline $\begin{array}{l}\text { Pardal et al, } \\
2016^{60}\end{array}$ & Right & Left UU & Not listed & USG, LS & $\begin{array}{c}\text { Ectopic } \\
\text { pregnancy }\end{array}$ & $\begin{array}{c}\text { Ruptured tubal } \\
\text { pregnancy }\end{array}$ & Salpingectomy \\
\hline $\begin{array}{l}\text { Gnanasambanthan } \\
\text { et al, } 2017^{13}\end{array}$ & Left & Right UU & Not listed & USG, LS & $\begin{array}{c}\text { Ectopic } \\
\text { pregnancy }\end{array}$ & $\begin{array}{c}\text { Ectopic } \\
\text { pregnancy }\end{array}$ & Salpingectomy \\
\hline $\begin{array}{l}\text { Kabashi et al, } \\
2016^{28}\end{array}$ & Right & None & Right RA & USG, MRI & $\begin{array}{c}\text { Paraovarian } \\
\text { cysts in RUO }\end{array}$ & None & None \\
\hline $\begin{array}{l}\text { Sinonquel et al, } \\
2018^{7}\end{array}$ & Right & Left UU & None & USG, CT LS & $\begin{array}{l}\text { Mucocele, } \\
\text { duplication }\end{array}$ & Right UO & None \\
\hline $\begin{array}{l}\text { Ireo et al, } \\
2018^{17}\end{array}$ & Right & Left UU & None & $\begin{array}{l}\text { HSG, USG } \\
\text { MRI, LS }\end{array}$ & Right UO & Right UO & $\begin{array}{c}\text { Salpingo- } \\
\text { oophorectomy }\end{array}$ \\
\hline $\begin{array}{l}\text { Bagga et al, } \\
2018^{36}\end{array}$ & Right & $\begin{array}{l}\text { Left UU, Upper } \\
\text { vaginal atresia }\end{array}$ & None & USG, LS & Haematometra & Right UO & Salpingectomy \\
\hline $\begin{array}{l}\text { Woolnough et al, } \\
2019^{59}\end{array}$ & Left & Right UU & Not listed & LS & $\begin{array}{c}\text { Ectopic } \\
\text { pregnancy }\end{array}$ & $\begin{array}{c}\text { Ruptured tubal } \\
\text { pregnancy }\end{array}$ & Salpingectomy \\
\hline $\begin{array}{l}\text { Jang et al, } \\
2020^{35}\end{array}$ & Right & Left UU & None & TVUS, LS & $\begin{array}{c}\text { Ectopic } \\
\text { pregnancy }\end{array}$ & $\begin{array}{c}\text { Ectopic } \\
\text { pregnancy }\end{array}$ & Salpingectomy \\
\hline $\begin{array}{l}\text { Wei et al, } \\
2020^{66}\end{array}$ & Bilateral & none & none & TVUS, LS & $\begin{array}{c}\text { Ectopic } \\
\text { pregnancy }\end{array}$ & $\begin{array}{c}\text { Ectopic } \\
\text { pregnancy }\end{array}$ & Salpingectomy \\
\hline $\begin{array}{l}\text { Kathopoulis et al, } \\
2021^{57}\end{array}$ & Right & Left UU & Not listed & USG, LS & $\begin{array}{c}\text { Ectopic } \\
\text { pregnancy }\end{array}$ & $\begin{array}{c}\text { Ectopic } \\
\text { pregnancy }\end{array}$ & Salpingectomy \\
\hline $\begin{array}{l}\text { Mariani et al, } \\
2021^{15}\end{array}$ & Left & Right UU & Left RA & $\begin{array}{c}\text { USG,TVUS } \\
\text { MRI }\end{array}$ & None & None & None \\
\hline
\end{tabular}

Abbreviations: UO, undescended ovary; UU, unicornuate uterus; RA, renal agenesis; USG, ultrasonography; TVUS, transvaginal ultrasonography; CT, computed tomography; IVP, intravenous pyelography; HSG, hysterosalpingography; MRI, magnetic resonance imaging; LS, laparoscopy; LT, laparotomy.

ovary". ${ }^{42}$ In 1933 Walker used the term of UO in a report where he presented a new case and also made reference to 2 other cases, one dating from $1896 .{ }^{43}$ We found a case report from 1965 by PC Bansal, who discovered an UO during a postmortem examination and 2 other reports about the same pathology made by Schumacher in 1938 and Schattenberg in $1940 .{ }^{44}$ Because of incomplete information we did not include these cases in our review.
The estimated incidence of UO is $0.3-2 \%,{ }^{1,8,14,45}$ although other authors consider it to be $0.3-0.5 \%{ }^{2,6,7,34}$ The low incidence might be due to underdiagnosis, since ectopic ovaries can be easily missed during routine investigations or are often asymptomatic. The incidence can reach up to $22 \%$ when congenital uterine anomalies are associated, ${ }^{1,9}$ to $20 \%$ in the presence of Mayer-RokitanskyKüster-Hauser syndrome (agenesis of the uterus, cervix and 
Table 3 Asymptomatic Cases in Adult Patients

\begin{tabular}{|c|c|c|c|c|}
\hline Authors, Year & Laterality & $\begin{array}{c}\text { Müllerian } \\
\text { Malformations }\end{array}$ & $\begin{array}{c}\text { Renal } \\
\text { Malformations }\end{array}$ & $\begin{array}{c}\text { Methods } \\
\text { of Diagnosis }\end{array}$ \\
\hline \multirow[t]{5}{*}{ Verkauf et al, $1999^{2}$} & Bilateral & Bicornuate uterus & None & HSG, LS \\
\hline & Left & None & None & HSG, HS, LS \\
\hline & Right & Left UU & None & HSG, LS \\
\hline & Right & None & None & HSG, LS \\
\hline & Right & None & None & HSG, LS \\
\hline Van Vorhis et al, $2000^{33}$ & Bilateral & Bicornuate uterus & None & USG, HSG, LS \\
\hline Görgen et al, $2002^{46}$ & Bilateral & Bicornuate uterus & None & TVUS, HSG, LS \\
\hline \multirow[t]{5}{*}{ Ombelet et al, $2003^{47}$} & Left & Right UU & None & HSG, MRI, IVP, LS \\
\hline & Left & Right UU & None & HSG, MRI, IVP, HS, LS \\
\hline & Left & Right UU & Left RA & HSG, MRI, LS \\
\hline & Right & Left UU & Right RA & HSG, MRI, IVP, LS \\
\hline & Right & Left UU & Not listed & USG, HSG, MRI \\
\hline Ombelet et al, $2003^{56}$ & Left & Right UU & None & HSG, USG, MRI, IVP, HS, LS \\
\hline \multirow[t]{2}{*}{ Ombelet et al, $2003^{45}$} & Left & Right UU & Left RA & HSG, MRI, LS \\
\hline & Right & Left UU & None & USG, MRI \\
\hline Halil et al, $2010^{18}$ & Right & None & Not listed & USG, LS \\
\hline Uyar et al, $201 \mathrm{I}^{37}$ & Right & Left UU & Right RA & USG, MRI, HSG, LS \\
\hline Ombelet et al, $20 \mathrm{II}^{3}$ & Right & Left UU & Right RA & HSG, USG, MRI, LS \\
\hline Shih et al, $2015^{16}$ & Bilateral & MRKH & Left RA & CT, LS \\
\hline Garg et al, $2016^{34}$ & Bilateral & None & None & TVUS, HSG, MRI, HS, LS \\
\hline Oride et al, $2016^{49}$ & Bilateral & Right UU, SU & None & USG, HSG, MRI, HS, LS \\
\hline Verma et al, $2018^{32}$ & Left & Right UU & None & HSG, USG, LS \\
\hline Mukherjee et al, $2020^{14}$ & Left & Right UU & Left RA & TVUS, HS, LS \\
\hline \multirow[t]{3}{*}{ Wei et al, $2020^{66}$} & Right & None & None & USG, HS \\
\hline & Bilateral & None & None & TVUS, HS \\
\hline & Left & None & None & LS \\
\hline Pereira et al, $2021^{55}$ & Right & Left UU & Not listed & HSG \\
\hline
\end{tabular}

Abbreviations: UU, unicornuate uterus; MRKH, Mayer-Rokitansky-Küster-Hauser syndrome; RA, renal agenesis; SU, septate uterus; USG, ultrasonography; TVUS, transvaginal ultrasonography; CT, computed tomography; IVP, intravenous pyelography; HSG, hysterosalpingography; MRI, magnetic resonance imaging; LS, laparoscopy; HS, hysteroscopy.

upper portion of the vagina), ${ }^{10}$ and to $42 \%$ when occurring in conjunction with controlateral UU. . $7,14,17,31,46$ Ombelet et al reported that $62.5 \%$ of patients with UU had also UO. ${ }^{47}$ The study of Wang et al reported a UO incidence of $28 \%$ in MRKH patients compared to $5 \%$ in females with normal uterus. ${ }^{10}$ The incidence of associated renal abnormalities is $23 \%$, the most frequent being ipsilateral renal agenesis, found by El-Bishri et al in 38\% of cases. ${ }^{48}$

The present literature research found 74 patients, including 12 children (16\%). In 55 cases there were associated 
Müllerian malformations: 1 septate uterus, 2 cervix atresia, 1 upper vaginal atresia, 2 MRKH, 5 bicornuate uterus, 47 contralateral UU. Nineteen patients $(25.6 \%)$ had a normal uterus. Renal malformations were not listed for 14 patients, but of the remaining 60 cases, $20(33.3 \%)$ had ipsilateral renal agenesis. In conclusion, $74.3 \%$ of cases had associated Müllerian malformations, with a $63.5 \%$ incidence of UU. Comparing the symptomatic patients (including the children) with the asymptomatic group, we found no significant differences in terms of Müllerian malformations $(76.6 \%$ versus $70.3 \%$ ) or renal agenesis $(27.6 \%$ versus $25.9 \%)$.

\section{Clinical Features and Differential Diagnosis}

An accurate diagnosis based on clinical findings is challenging because of the nonspecific signs and symptoms mimicking other abdomino-pelvic pathologies. There are asymptomatic and symptomatic forms. Asymptomatic UO is usually discovered during infertility work-up. ${ }^{43,49,50}$ Verma et al, in 2018, found 30 reported cases, of which $60 \%$ were asymptomatic and $40 \%$ symptomatic. $^{32}$ Our literature research found 47 symptomatic cases $(63.5 \%)$ : $12 / 12$ children $(100 \%)$ and $35 / 62$ adult patients $(56.4 \%)$. In total, there were $77 \%$ symptomatic UO and $23 \%$ asymptomatic forms.

Some anamnestic key features could direct the clinician to an ovarian malposition: cyclic abdominal pain above the pelvis (due to cyst formation or ovulation), dysmenorrhea or menstrual irregularities, associated uterine or renal malformations or a history of infertility. $8,12,18,32$ When complications such as torsion, cyst rupture or ectopic tubal pregnancy occur, they may lead to acute surgical abdomen., 5,15

In terms of differential diagnosis, the most common are appendicular pathologies (acute appendicitis, mucocele) or gynecological disorders. When abdominal pain is located above the pelvis, the differential diagnosis could be intestinal duplication or tumors, acute cholecystitis, nephrolithiasis or pyelonephritis. $5,6,8$

As can be seen in Table 1, 11 (91.6\%) pediatric cases were diagnosed by surgical exploration; 7 (63.6\%) were laparotomies. In the group of symptomatic adult patients (Table 2), 31 (88.5\%) were diagnosed by surgical exploration, of which 13 (41.9\%) were laparotomies. In terms of differential diagnosis and considering a total of 47 symptomatic patients, an ectopic pregnancy was suspected in 15 $(31.9 \%)$ cases. Other situations of misdiagnosis include 5 acute appendicitis, 4 intestinal duplications, 3 appendiceal mucocele, 2 renal cysts and 1 acute cholecystitis.

\section{Imaging Diagnosis}

Ovarian maldescent may be unilateral or bilateral, but it has been reported to be more common on the right side and more likely to be located retroperitoneal. ${ }^{33,34}$

Our literature research found 39 right UO (52.8\%), 23 left UO (31\%), and 12 bilateral UO (16.2\%). We realised a comparative analysis of the laterality and we discovered that the incidence of the right UO is $59.5 \%$ for the symptomatic group (66.6\% in children and $57.1 \%$ in adults) and $40.7 \%$ for the asymptomatic group (with $33.3 \%$ left UO). Acute pain in the right hemiabdomen worries more than the left one (appendicitis, cholecystitis), so patients request a medical investigation more often in these circumstances, which may lead to a higher incidence of the right laterality. We hypothesize that the real right-left frequency is reflected by the asymptomatic group.

From the gross appearance point of view, in most instances the $\mathrm{UO}$ is elongated in its longitudinal axis and often with microcysts. ${ }^{2}$ The infundibulopelvic ligament is shortened while the utero-ovarian ligament is elongated. ${ }^{2,34}$ When UU is associated, the fallopian tube on the same side as the UO may have different features. It may not be attached to the uterus and only the infundibulum and the fimbriae develop attached to the undescended ovary. It also can be found to be rudimentary or of normal aspect but elongated. $2,24,30,31,35$

The first option in imaging investigation is the traditional 2D ultrasound scan, which allows the detection of uterine and renal malformations and may even find a maldescended ovary. ${ }^{8,15} 3 \mathrm{D}$ ultrasonography or transvaginal ultrasound (in sexually active patients) may provide additional information. However, in pediatric patients it is well known that even experienced radiologists can have difficulties visualizing the ovaries on abdominal USG (agitated or scared patients, uncooperative patients, small ovaries, empty bladder). Consequently, if the ovary could not be found in its normal location, especially when associated with Müllerian abnormalities, efforts should be made to locate it.

Among further explorations, magnetic resonance imaging (MRI) represents the first choice, being traditionally thought the most sensitive imaging modality for evaluation of Müllerian and urinary structures. In addition, it provides multiplanar images, clear anatomical detail and tissue characterization without ionizing radiation, which is 
extremely suitable for children and pregnant women., ${ }^{9,10}$ During investigations for infertility some cases are detected by HSG or salpingoscopy. ${ }^{34,49,51}$

Ultimately, an accurate diagnosis is made by laparotomy or laparoscopy, despite all the advanced imaging techniques and especially in cases of acute abdomen. Laparoscopy is considered nowadays the gold standard for the diagnosis of UO. However, Ombelet et al suggested performing routine MRI after ovarian stimulation with clomiphene citrate as a better tool than laparoscopy in the diagnosis of UO due to lower costs, non-invasivity and detection of retroperitoneal structures. ${ }^{47}$

During the present literature research, we found that the following imaging techniques were used to provide an accurate diagnosis: 42 ultrasound investigations, 24 MRIs, 7 CTs, 25 HSG, 8 HSs, 12 IVPs, 20 laparotomies and 43 laparoscopies (22 in asymptomatic cases). HSG and HS were used mostly for women with a history of infertility.

\section{Evolution and Prognostic Concerns}

The evolution of the UO should be identical to a normally positioned one, with ovulation but also with complications such as cyst formation, cyst ruptures or tumors. Our literature research found 1 torsion, ${ }^{38} 1$ functional cyst, ${ }^{12} 1$ paraovarian cyst, ${ }^{31} 4$ ruptures of hemorrhagic cysts,, 5,6,30,49 and 1 case of pyosalpinx. ${ }^{40}$

The retroperitoneal position of the ovary may lead to a higher risk of malignant changes in analogy with the undescended testis. ${ }^{4,16,17,52}$ After searching the literature we found 4 cases of cystadenomas in UO, including our patient, but no malignant neoplasms. ${ }^{38,53,54}$ Thus we can conclude that there is no documented association between UO and malignancy.

Another unclear relationship is between the UO and infertility. There is strong evidence of retrieving oocytes from an ectopic ovary, ${ }^{18,33,55}$ as well as of a spontaneous intrauterine pregnancy with a transperitoneal migrated oocyte produced from a UO. ${ }^{56,57}$ In 2016 Suh postulated that, for a spontaneous pregnancy to occur, a patient needs to possess at least 1 normal tube and 1 functional ovary. ${ }^{5}$ A dreaded complication is represented by the ectopic pregnancy in an undescended fallopian tube (UFT). In these specific cases, ectopic tubal pregnancies seem to be linked to the Müllerian malformations not to the UO per se. ${ }^{58-60}$ There is a $22 \%$ incidence for ectopic pregnancy in a UU with rudimentary horn, but only $4 \%$ is the estimated risk of ectopic pregnancy in patients with isolated UO. ${ }^{5,8,48}$ Thus, with a normal uterus, even with one UO, the pregnancy could evolve normally.

Among the 74 cases we found in the literature, there were 16 ectopic pregnancies $(21.6 \%)$, with only 4 complicated cases (ruptured ectopic pregnancy). Except in 1 case, all of these patients had contralateral unicornuate uterus.

\section{Principles of Treatment}

Because the UO is functional and contains a corpus luteum, ${ }^{12,13}$ conservative treatment of this condition is recommended by all the authors. Even incidentally discovered intraoperative UO should not be treated differently than a normally situated ovary. ${ }^{7,61}$

Surgical treatment remains indicated for the correction of associated Müllerian malformations (uterine septum, cervical or vaginal atresia), for complications related to UO (torsion, cyst rupture, tumor) and for ectopic pregnancy in the UFT. Many authors suggested that, once recognized, a non-communicating UFT should be removed in order to reduce the risk of ectopic pregnancy and to eliminate the competition between the tubes for the transperitoneal migrating sperm or oocyte. ${ }^{48,62-64}$ Whenever surgical management is needed, the final aim is to preserve the ovary. As for a normally positioned ovary, uncomplicated simple cysts smaller than $50 \mathrm{~mm}$ can be treated in such a way as to conserve the ovary, ovariectomy remaining indicated only for isolated cases such as necrotic torsioned ovary or malignant features. However, we found 2 reported cases of UO with recurrent pain, where patients decided to excise the malpositioned but otherwise normal ovary. $^{17,65}$

As alternative surgical interventions, we found in-situ fixation of the UO for preventing torsion, ${ }^{11}$ or its transposition to the posterior wall of the uterus when the other ovary is absent or does not respond to ovarian hyperstimulation treatment. ${ }^{18}$ In patients with persistent recurrent abdominal pain, denervation of the ovary or ovarian suppression with oral contraceptives were suggested. ${ }^{30,31}$

This literature research revealed that oophorectomy was made in 11 cases: $5(41.6 \%)$ in children and 6 $(17.1 \%)$ in adult symptomatic women. Other surgical interventions were: 4 cystectomies and 13 salpingectomies (11 for ectopic tubal pregnancies, 2 for unaffected undescended tubes). None of the asymptomatic patients (discovered during infertility investigations) had a surgical technique involving the ovary or the tube. Through more detailed research, we found 6 children and 5 women with pathological UO (mainly cystic lesions), so it seems that, 
unlike the cases of cystic normally located ovaries, nonconservative management was preffered.

\section{Practical Applicability: Case Illustration}

A 14-year-old premenarcheal girl was referred to our clinic with abdominal pain in the right iliac fossa and flank, followed by nausea and inappetence. Pains had started 3 months ago, but they had worsened 2 days before presentation. On clinical examination, we found tenderness in the right hemiabdomen, without signs of renal pathology. Our first diagnosis was acute appendicitis but the routine laboratory tests were normal. The USG showed in the right flank an anechoic $55 \mathrm{~mm} / 40 \mathrm{~mm}$ cystic tumor, well-defined by hyperechoic borders. The uterus seemed to be developed according to age, but ovaries could not be accurately documented. Our second diagnosis was right renal cystic dysplasia or intestinal duplication. We requested an abdominal MRI, which showed in the right flank a 55mm/40mm-sized mass, with liquid content, welldefined, having in the periphery a few cystic images (Figures 2 and 3). The left kidney was normal. However, although the uterus had a left unicornuate aspect, an ovarian-like structure was described in the right side of the pelvis.

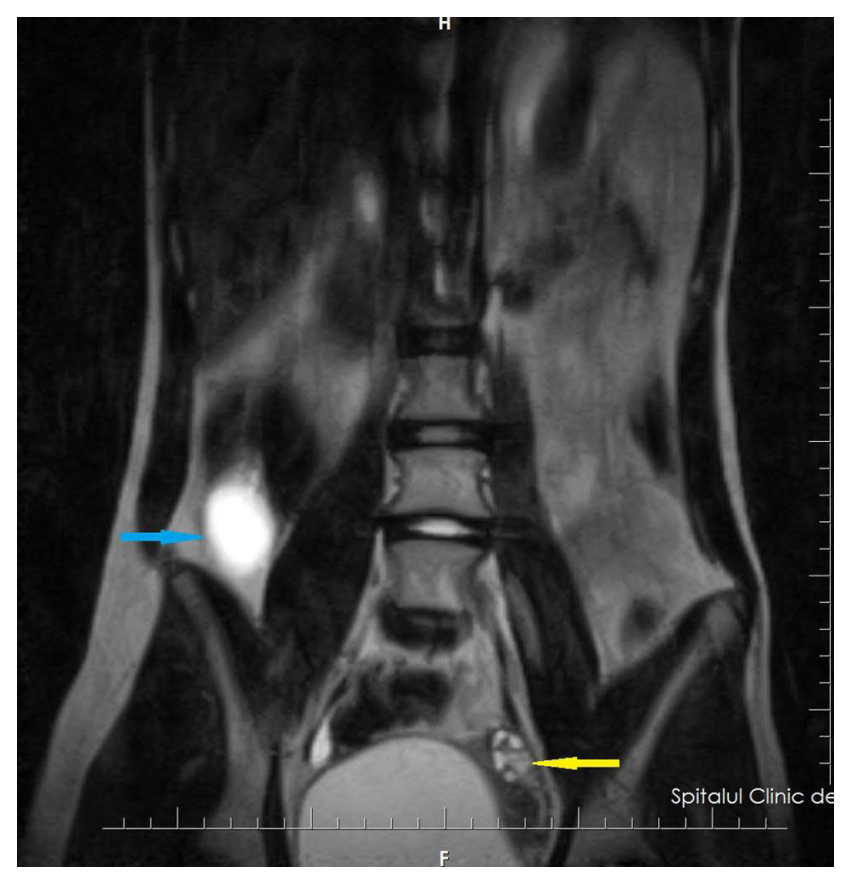

Figure 2 T2 cor MRI. Blue arrow: cystic tumor; yellow arrow: left ovary.

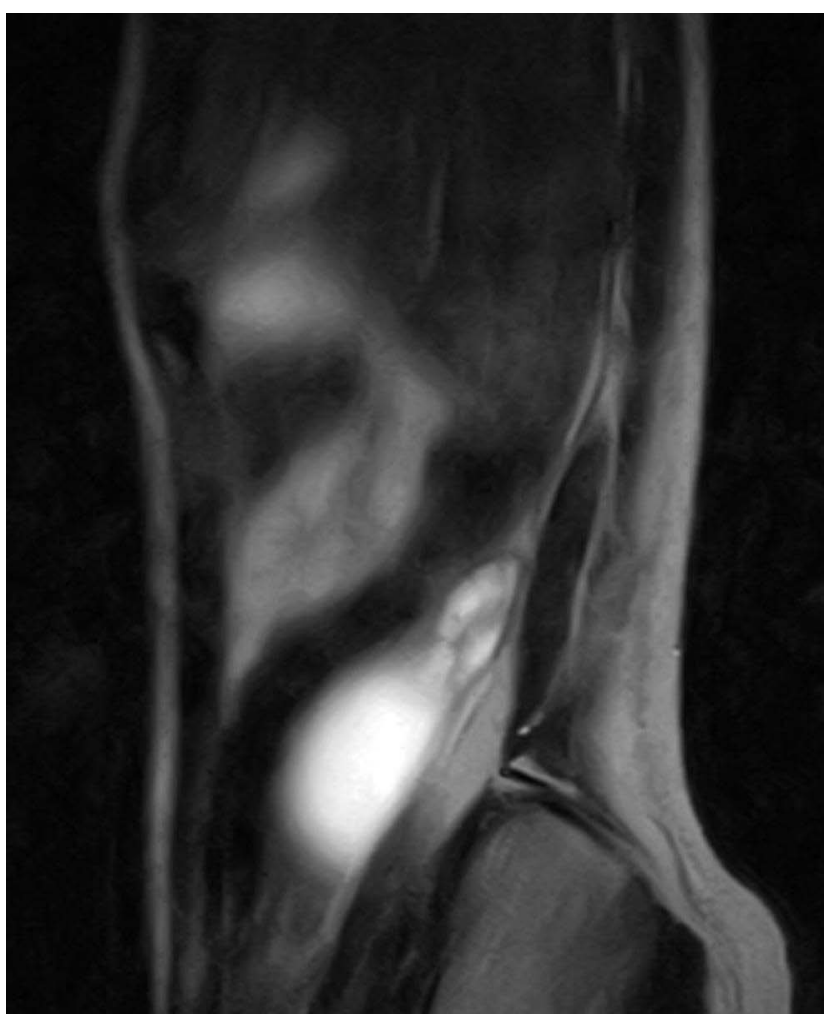

Figure 3 Cystic tumor on T2 sag MRI.

After a thorough debate with our radiologists, we established the diagnosis of retroperitoneal cyst, probably of renal origin, and proceeded to surgical intervention. After a median laparotomy we found retroperitoneal in the right flank, a $55 \mathrm{~mm} / 40 \mathrm{~mm}$ cystic tumor, with a wide insertion onto the posterior abdominal wall, presenting at the upper pole an area of tissue suggestive of the ovary and a distal fimbriated structure suggesting the fallopian tube without contact with the uterus. The appendix and the right colon were of normal appearance. The uterus was unicornuate on the left side, with normal left tube and ovary. Neither the right kidney nor the ureter were visualized. We agreed that the tumor was in fact an undescended ovarian cyst, but because there were dilated vessels on the surface and a little solid area inside, we decided to perform a right salpingo-oophorectomy. Histopathological sections showed a serous cystadenoma with a non-communicating fallopian tube. Postoperatively, the recovery was uneventful and the patient was discharged from the hospital one week later.

\section{Conclusions}

The low incidence of UO might be due to underdiagnosis, which confirms that "the eyes do not see what the mind does 
not know". UO should be considered in the differential diagnosis of acute or cyclical abdominal pain regardless of age, as well as in cases of infertility, especially in patients with an absent ovary on the routine USG and with associated renal or Müllerian malformations. MRI with or without clomiphene citrate ovarian stimulation is the best non-invasive tool for a correct diagnosis, while the laparoscopy remains the gold standard. Because there is no reported increased incidence of malignancy in UO per se, the treatment should be conservative in order to spare a malpositioned but otherwise normal functioning ovary. Of great importance is counseling patients with an emphasis on the fact that UO is a developmental condition rather than a malformation, but with an increased risk of infertility or ectopic pregnancy.

\section{Abbreviations}

UO, undescended ovary; USG, ultrasonography; HSG, hysterosalpingogram; MRI, magnetic resonance imaging; UU, unicornuate uterus; UFT, undescended fallopian tube.

\section{Data Sharing Statement}

The datasets are available from Dr Liliana Baroiu on reasonable request.

\section{Ethics Approval and Informed Consent}

Ethics approval was obtained from the Ethics Commission of the Clinical Emergency Hospital for Children "Sf. Ioan”, Galaţi, Romania (approval no: 997/January 29, 2019).

\section{Consent for Publication}

Written informed consent was obtained from the patient prior to publication.

\section{Acknowledgments}

The present work was academically supported by 'Dunarea de Jos' University of Galati, through the research center Multidisciplinary Integrated Center of Dermatological Interface Research (MIC-DIR).

\section{Author Contributions}

All authors made substantial contributions to conception and design, acquisition of data, or analysis and interpretation of data; took part in drafting the article or revising it critically for important intellectual content; agreed to submit to the current journal; gave final approval of the version to be published; and agree to be accountable for all aspects of the work.

\section{Funding}

The present study was supported by the 'Dunarea de Jos' University of Galati through an internal grant (grant no. RF3668/01.10.2021).

\section{Disclosure}

The authors declare that they have no financial competing interests.

\section{References}

1. Dabirashrafi H, Mohammad K, Moghadami-Tabrizi N. Ovarian malposition in women with uterine anomalies. Obstet Gynecol. 1994;83 (2):293-294.

2. Verkauf BS, Bernhisel MA. Ovarian madescent. FertilSteril. 1996;65 (1):189-192.

3. Ombelet W, Verswijvel G, Vanholsbeke C, Schobbens JC. Unicornuate uterus and ectopic (undescended) ovary. Facts Views Vis Obgyn. 2011;3(2):131-134.

4. Trinidad C, Tardáguila F, Fernández GC, Martínez C, Chávarri E, Rivas I. Ovarian maldescent. Eur Radiol. 2004;14(5):805-808. doi:10.1007/s00330-003-2202-3

5. Suh DS, Han SE, Yun KY, Lee NK, Kim KH, Yoon MS. Ruptured hemorrhagic corpus luteum cyst in an undescended ovary: a rare cause of acute abdomen. J Pediatr Adolesc Gynecol. 2016;29 (1):21-24. doi:10.1016/j.jpag.2015.09.004

6. Sekmenli T, Gündüz M, Ciftci I. Ruptured hemorrhagic cyst of undescended ovary mimicking mucocele: a rare pediatric case. Iran J Med Sci. 2017;42(1):98-101.

7. Sinonquel P, Bontinck J, Stevens M. Undescended ovary and fallopian tube presenting as appendiceal mucocele. Facts Views Vis Obgyn. 2018;10(1):47-50.

8. Dietrich JE, Hertweck SP, Bond S. Undescended ovaries: a clinical review. J Pediatr Adolesc Gynecol. 2007;20:57-60. doi:10.1016/j. jpag.2006.12.007

9. Allen JW, Cardall S, Kittijarukhajorn M, Siegel LC. Incidence of ovarian maldescent in women with mullerian duct anomalies: evaluation by MRI. AJR Am J Roentgenol. 2012;198(4):W381-5. doi:10.2214/AJR.11.6595

10. Wang Y, Lu J, Zhu L, et al. Increased incidence of abnormally located ovary in patients with Mayer-Rokitansky-Küster-Hauser syndrome: a retrospective analysis with magnetic resonance imaging. AbdomRadiol (NY). 2018;43(11):3142-3146.

11. Altinay-Kirli E, Özcan R, Öncül M, et al. A rare cause of abdominal pain: ectopic ovary and intestinal malrotation. Turk $J$ Pediatr. 2017;59(6):699-703. doi:10.24953/turkjped.2017.06.014

12. Cohen JA, Holzman MD. A giant ectopic ovary. J Laparoendosc Adv Surg Tech A. 2001;11(1):31-35. doi:10.1089/10926420150502913

13. Gnanasambanthan S, Uchil D. A rare case of an ectopic pregnancy in the rudimentary tube of a patient with an isolated unicornuate uterus. BMJ Case Rep. 2017. doi:10.1136/bcr-2017-220825

14. Mukherjee S, Sanghamita R, Nandita B. Ovarian maldescent: a case report and discussion. Int $J$ Reprod Contracept Obstet Gynecol. 2020;9(5):2164-2166. doi:10.18203/2320-1770.ijrcog20201826

15. Mariani L, Mancarella M, Cirillo S, Biglia N. Unicornuate uterus associated with retrosigmoid ovary and unilateral renal agenesis: a case report and a review of the literature. Eur Gynecol Obstetrics. 2021;3(2):72-75. 
16. Shih TH, Ho CM. Undescended ovary mimicking appendiceal mucocele. Surgery. 2015;157(4):824-825.

17. Ireo E, Haruna M, Gandhi P. Laparoscopic management of maldescended ovary presenting with recurrent acute abdomen. Gynecol Minim Invasive Ther. 2018;7(2):74-77. doi:10.4103/GMIT. GMIT_16 18

18. Halil S, Kucuk M, Çepni IL. Surgical transpositioning of ectopic ovarian tissue and pregnancy. GynecolObstet Invest. 2010;69:78-80.

19. Wharton L. Two cases of supernumerary ovary and one of accessory ovary, with an analysis of previously reported cases. Am J Obstet Gynecol. 1959;78:1101-1119. doi:10.1016/S0002-9378(16)36660-1

20. Lachman M, Berman M. The ectopic ovary. A case report and review of the literature. Arch Pathol Lab Med. 1991;115:233-235.

21. Kusaka M, Mikuni M. Ectopic ovary: a case of autoamputated ovary with mature cystic teratoma into the cul-de-sac. $J$ ObstetGynaecol Res. 2007;33:368-370.

22. Bayramov V, Şükür YE, Çetinkaya E, Berker B. Ectopic ovary autotransplanted over rectosigmoid colon: a case report. Fertil Steril. 2009;92:1496.e15-1496.e16.

23. Rock JA, Parmley T, Murphy AA, Jones HW Jr. Malposition of the ovary associated with uterine anomalies. Fertil Steril. 1986;45 (4):561-563.

24. Brown C, LaVigne WE, Padilla SL. Unruptured pregnancy in a heterotopic fallopian tube: evidence for transperitoneal sperm migration. Am J Obstet Gynecol. 1987;156(1):88-90. doi:10.1016/ 0002-9378(87)90211-0

25. Sadler TW. Urogenital system. In: Kluwer W, editor. Langman's Medical Embryology. 14th ed. Philadelphia; 2019:267-272.

26. Schoenwolf GC, Bleyl SB, Brauer PR, Francis-West PH. Development of the urogenital system. In: Churchill Livingstone, editor. Larsen's Human Embryology. 4th ed. New York; 2009: 515-519.

27. Jacquinet A, Millar D, Lehman A. Etiologies of uterine malformations. Am J Med Genet A. 2016;170:2141-2172. doi:10.1002/ajmg.a.37775

28. Kabashi S, Dedushi K, Mucaj S, Ramadani N, Shatri J, Hoxhaj A. True ectopia of right ovary with ipsilateral renal agenesis. $J$ Med Cases. 2016;7(9):373-375. doi:10.14740/jmc2494w

29. Kollia P, Kounoudes C, Veloudis G, Giannakou N, Gourgiotis S. True ectopic ovary in the right iliac fossa mimicking acute appendicitis and associated with ipsilateral renal agenesis. J Obstet Gynecol Res. 2014;40(3):858-861.

30. Kives SL, Perlman S, Bond S. Ruptured hemorrhagic cyst in an undescended ovary. J Pediatr Surg. 2004;39(11):44-46. doi:10.1016/j.jpedsurg.2004.07.030

31. Desteli GA, Gursu T, Cevik H, Zeyneloglu HB. Undescended ovary presented with paraovarian cyst. J Turk Soc Obstet Gynecol. 2013;10 (4):272-274. doi:10.5505/tjod.2013.59862

32. Verma S, Jindal UN, Joshi B. Ovarian maldescent in unicornuate uterus; diagnostic and therapeutic challenge. $J$ Gynecol Women's Health. 2018;10(5):555800.

33. VanVoorhis BJ, Dokras A, Syrop CH. Bilateral undescended ovaries: association with infertility and treatment with IVF. FertilSteril. 2000;74(5):1041-1043.

34. Garg D, Grazi R, Kankanala N, Melzer-Ross K. Bilateral ovarian maldescent: unusual cause of infertility: a case report and literature review. J ObstetGynaecol Res. 2016;42(8):1038-1041.

35. Jang YS, Kim YS. A case report of ectopic pregnancy arising in a unicornuate uterus, accompanied by the undescended tube and ovary with double inferior vena cava. Medicine (Baltimore). 2020;99(28):e21105. doi:10.1097/MD.0000000000021105

36. Bagga R, Muthyala T, Saha PK, et al. left uterine horn with cervicovaginal atresia and ovarian maldescent - an unclassified Müllerian anomaly treated with horn-vaginal anastomosis. J Obstet Gynaecol. 2018;38(8):1176-1178.
37. Uyar I, Gulhan I, Sipahi HHM, Ozeren M. Ectopic ovary confirmed by ovarian stimulation in a case of unicornuate uterus. Fertil Steril. 2011;96(2):122-124.

38. Adnopoz A, Davis JG. Torsion of an ectopic ovarian cyst complicating early pregnancy. Discussion of unusual anatomy and etiology. AMA Arch Surg. 1951;62(2):292-294. doi:10.1001/archsurg.1951.01250030297011

39. Rakhshankhah N, Ebrahimi R, AtaeeKachuee M, et al. A very rare case report of bilateral maldescended ovaries and müllerian duct anomaly associated with inflammatory myositis, myasthenia gravis and thymic pathology. Egypt J Radiol Nucl Med. 2021;52:254.

40. Tatu AL, Ionescu MA. Multiple autoimmune syndrome type IIIthyroiditis, vitiligo and alopecia areata. Acta Endo (Buc). 2017;13 (1):124-125. doi:10.4183/aeb.2017.124

41. Ciobotaru OR, Lupu MN, Rebegea L, et al. DexamethasoneChemical structure and mechanisms of action in prophylaxis of postoperative side effects. REV CHIM (Bucharest). 2019;70(3):843-847. doi:10.37358/RC.19.3.7017

42. Evans A, Cade S. Ectopic ovary. B J Surg. 1926;13(49):182-183. doi:10.1002/bjs. 1800134924

43. Walker RM, Lond MS. A case of undescended ovary. The Lancet. 1933;222(5748):972. doi:10.1016/S0140-6736(01)18962-2

44. Bansal PC. A case of unicornuate uterus and undescended left ovary. J Obstet Gynecol India. 1965;3:131.

45. Ombelet W, Verswijvel G, de Jonge E. Ectopic ovary and unicornuate uterus. $N$ Engl J Med. 2003;348(7):667-668. doi:10.1056/ NEJM200302133480722

46. Görgen H, Api M, Delikara N. Undescended fallopian tubes and ovaries: a rare incidental finding during an infertility investigation work up. Acta Obstet Gynecol Scand. 2002;81:371-374.

47. Ombelet W, Grieten M, DeNeubourg P, et al. Undescended ovary and unicornuate uterus: simplified diagnosis by the use of clomiphene citrate ovarian stimulation and magnetic resonance imaging (MRI). Hum Reprod. 2003;18(4):858-862. doi:10.1093/humrep/deg191

48. El-Bishry G, Faraj R, Tselos E. Sub-hepatic ectopic pregnancy in a fallopian tube of a non-communicating rudimentary uterine horn. Gynecol Surg. 2006;3:289-292. doi:10.1007/s10397-006-0214-0

49. Oride A, Kanasaki H, Ishihara T, Kyo S. Rare case of complicated congenital anomalies of female reproductive organs with bilateral undescended ovaries. J ObstetGynaecol Res. 2016;42(7):895-898.

50. Mahmoud MS. Maldescended ovary associated with Müllerian and kidney dysgenesis: a case report. J Reprod Med. 2014;59(56):337-340.

51. Ombelet W, Stroef F, Grieten M, Verswijvel G, Hinoul P, de Jonge E. Unicornuate uterus and undescended ovary: diagnostic and therapeutic implications. J ObstetGynecol India. 2005;55(4):339-344.

52. Nichols D, Postoloff AV. Congenital ectopic ovary. Am J Obstet Gynecol. 1951;62(1):195-198. doi:10.1016/0002-9378(51)91111-8

53. Das D, Dhingra $S$ An unusual case of serous cystadenoma in undescended right ovary: a case report. Poster presented at RCOG World Congress;March 28-30; Hyderabad, India;2014.

54. Campbell BF, Julian TM. Bilateral retroperitoneal ovaries. A case report. J Reprod Med. 1988;33(10):839-842.

55. Pereira N, Willson S, McCarter K, Chung PH, Kligman I, Rosenwaks Z. Combined transvaginal and transabdominal oocyte retrieval in a patient with an ectopic ovary and unicornuate uterus. Fertil Steril. 2021;115(5):1347-1349.

56. Ombelet W, Deblaere K, Grieten M, et al. Intrauterine pregnancy following transperitoneal oocyte and/or sperm migration in a woman with an ectopic (undescended) ovary. Reprod Biomed Online. 2003;7 (1):110-113. doi:10.1016/S1472-6483(10)61738-5

57. Kathopoulis N, Kypriotis K, Chatzipapas I, Samartzis K, Zacharakis D, Protopapas A. Ectopic pregnancy in a hypoplastic fallopian tube at an extrapelvic location. J Minim Invasive Gynecol. 2021;28(6):1133-1134. doi:10.1016/j.jmig.2020.11.027 
58. Nielsen KG, Pedersen AT. Rare disease: an ectopic pregnancy under the liver. BMJ Case Rep. 2010;2010(sep23 1):bcr0120102615bcr0120102615. doi:10.1136/bcr.01.2010.2615

59. Woolnough B, Ballermann C. An atypical presentation of ectopic pregnancy with unicornuate uterus and undescended fallopian tube. J Obstet Gynaecol Can. 2019;41(2):214-216.

60. Pardal C, Braga L, Vides B. Ectopic pregnancy in an undescended fallopian tube: a diagnostic challenge. BMJ Case Rep. 2016; bcr2015213058. doi:10.1136/bcr-2015-213058

61. Watkins BP, Kothari SN. True ectopic ovary: a case and review. Arch Gynecol Obstet. 2004;269:145-146. doi:10.1007/s00404-003-0554-1

62. Timmerman DJ, Lewis BV. Laparoscopic treatment of an ectopic pregnancy in an undescended fallopian tube. $J$ ObstetGynaecol. 1993;13(4):291-292.

63. Knudsen HJ, Clausen IH. An extra-uterine pregnancy in an ectopic fallopian tube. Zentralbl Gynakol. 1994;16(9):544-545.
64. Pokoli TB. Ectopic pregnancy in a noncommunicating tube of a unicornuate uterus. A case report. J Reprod Med. 1989;34 (12):994-995.

65. Mama ST. Ectopic ovary presenting as right upper quadrant abdominal pain in a premenarchal patient with a unicornuate uterus. $J$ Pediatr Adolesc Gynecol. 2012;5(2):54. doi:10.1016/j. jpag.2011.12.015

66. Wei W, Luo W, Hu Q, Zeng L, Tang H, Wu R. Dysplasia of ovary without abnormal development of uterus and urinary system: a report of four cases. J Ovarian Res. 2020. doi:10.21203/rs.3.rs-58010/v1

67. Liberati A, Altman D, Tetzlaff J, et al. The PRISMA statement for reporting systematic reviews and meta-analyses of studies that evaluate health care interventions: explanation and elaboration. Journal of clinical epidemiology. 2009;62(10).

\section{Publish your work in this journal}

The International Journal of Women's Health is an international, peerreviewed open-access journal publishing original research, reports, editorials, reviews and commentaries on all aspects of women's healthcare including gynecology, obstetrics, and breast cancer. The manuscript management system is completely online and includes a very quick and fair peer-review system, which is all easy to use. Visit http://www.dovepress.com/testimonials.php to read real quotes from published authors. 